\title{
Present-Day Research Opportunities with Quasimonochromatic $\gamma$ Beams
}

\author{
D.L. Balabanski \\ Extreme Light Infrastructure - Nuclear Physics (ELI-NP), Horia Hulubei \\ National Institute for R\&D in Physics and Nuclear Engineering (IFIN-HH), \\ 30 Reactorului Str., 077125 Magurele, Romania
}

Received 23 October 2021

doi: https://doi.org/10.55318/bgjp.2021.48.5-6.597

\begin{abstract}
An overview of the main directions of present-day studies with quasimonochromatic $\gamma$ beams are discussed with an emphasis on the research opportunities which will be offered at the Extreme Light Infrastructure Nuclear Physics (ELI-NP) facility at Magurele near Bucharest in Romania. Experiments with $\gamma$ beams at the extremes of high temperature and high neutron-to-proton ratios are outlined. Some of the experimental setups for nuclear structure, reaction and astrophysics studies, which are available at ELI-NP, are described. Perspectives for studies of exotic nuclei within the Gamma Factory experiment at CERN are discussed. The expected fission-fragment yields at the Gamma Factory are presented. The perspectives for nuclear structure studies with $\gamma$ rays with orbital angular momentum are outlined.
\end{abstract}

KEY WORDS: active-target experiments, ion trapping, ion transport, multidetector arrays, nuclear astrophysics, nuclear structure, photonuclear reactions, photofission, quasimonochromatic $\gamma$ beams, twisted photons.

\section{Introduction}

In a recent review [1], the status and perspectives of photonuclear research were discussed, covering both, fundamental science and applications. The development of quasimonochromatic $\gamma$ beams with sufficient intensity, based the on the laser Compton backscattering (LCB) technique [2,3], opened the avenue for detailed studies of lowest-lying excitation modes in atomic nuclei, i.e., E1, M1 and E2 excitations. In pioneering experiments, Ohgaki et al. [4] and Pietralla et al. [5] used a polarized LCB $\gamma$ beam and demonstrated that nuclear dipole response can be studied at a new level of precision and sensitivity. Thus, experiments with LCB $\gamma$ beams turn out to be the ideal tool for studies of largeamplitude collective excitations in atomic nuclei, such as $e . g$., the isovector giant 


\section{D.L. Balabanski}

dipole resonance (IVGDR) or the pigmy dipole (PDR) and quadrupole (PQR) resonances. Here, the perspectives for nuclear resonance fluorescence (NRF) experiments and studies of dipole strength above the particle-evaporation threshold at ELI-NP are discussed.

Another application of intense $\gamma$-ray beams is related to photofission, a process in which ions of exotic neutron-rich isotopes are produced. Present-day photonuclear studies address measurements of photofission product yields [6,7], and kinetic energy, mass, charge and angular momentum distributions of fission fragments $[8,9]$. Photofission is also utilized for the production of beams of neutron-rich isotopes [10]. In this paper, the opportunities for production of rare isotope beams (RIBs) within the Gamma Factory experiment [11] at CERN are discussed, as well as the current technological developments which are under investigation at ELI-NP for the efficient transport and extraction of ions of exotic neutron-rich isotopes.

Laser beams of twisted photons carry out angular momentum higher than $1 \hbar$. For a recent review see, e.g., Ref. [13]. The generation of high-energy twisted photons would provide new diverse opportunities for nuclear structure research. Examples of possible experiments with high orbital angular momentum (OAM) $\gamma$ rays are highlighted.

An important aspect of present-day photonuclear physics are studies of photonuclear reactions. Photon-induced charged-particle reactions are related to nuclear astrophysics. Measurements of photoneutron reaction cross sections address issues such as radiation transport calculations $[14,15]$ or astroparticle problems like, e.g., ultra-high energy cosmic rays (UHECR) $[16,17]$. The perspectives for such studies together with the instruments, which are constructed at ELI-NP, are discussed. An important development in the field is the utilization of active targets. The program for active-target experiments and the detectors, which are under construction at ELI-NP, are presented.

\section{Nuclear Structure Studies at Extremely High Nuclear Temperatures}

Traditionally, photonuclear excitations were used for studies at the extreme of high nuclear temperatures. The interaction of photons with atomic nuclei is a very selective process. They induce dipole and, to a much lesser extend, quadrupole excitations due to the angular momentum selection rules. This allows to investigate dipole and quadrupole excitations over a large range of energies. Studies below and above the particle evaporation threshold are performed. In NRF experiments, it is possible to tune the quasimonochromatic $\gamma$ beam such that a discrete nuclear level is excited and study its decay. For details of the NRF formalism and methodology see Ref. [1]. Above the particle threshold, states in the continuum are excited. The deexitation goes either through emission of $\gamma$ rays, evaporation of particles, or fission. 


\section{Research with Quasimonochromatic $\gamma$ Beams}

Two detector arrays were constructed at ELI-NP for such studies. The ELI-NP

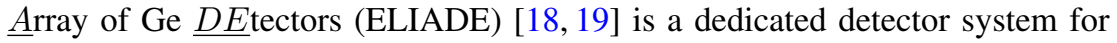
NFR experiments with quasimonochromatic $\gamma$ beams. It is composed of eight segmented HPGe Clover detectors, organized in two rings, at $90^{\circ}$ and $135^{\circ}$ with respect to the beam axis. The detectors are placed in the horizontal and vertical planes with respect to the beam. In addition, it is possible to add four largevolume $\mathrm{CeBr}_{3}$ detectors. At a later stage, the Clover detectors will be placed in anti-Compton shields. The overall efficiency of the array is about $6 \%$. This allows to measure $\gamma$-ray energies, intensities, angular distributions and polarization asymmetries, as well as $\gamma \gamma$ coincidences. Thus, it will be possible to extract from the measured spectra the $\gamma$-ray branching ratios, the $\gamma$-ray multipolarities and mixing ratios, the level energies, the spins, parities and the $K$ quantum numbers of the levels, their partial and total decay widths, the transition and excitation strength, and the integrated $\left(\gamma, \gamma^{\prime}\right)$ cross section. The performance of the ELIADE detectors was tested in-beam and the results meat the requirements for high-precision NRF experiments [20].

The family of $\underline{E L I}$-NP $\underline{G}$ amma $\underline{A}$ bove the $\underline{N}$ eutron $\underline{T h r e s h o l d ~(E L I G A N T) ~}$ instruments (see also Section 5.1) will be used to study $\left(\gamma, \gamma^{\prime}\right),(\gamma, \mathrm{n}),\left(\gamma, \gamma^{\prime} \mathrm{n}\right)$ and $(\gamma, \nu \mathrm{n}), \nu=1,2,3 \ldots$ reactions. For example, photoneutron cross sections will be measured with the ELIGANT - $\underline{T}$ hermal $\underline{N}$ eutron (ELIGANT-TN) setup [21], while the ELIGANT - $\underline{G}$ amma and $\underline{N}$ eutron (ELIGANT-GN) detector array [21,22] will be used for measurements of $\gamma$ rays and neutrons emitted during the deexcitation PDR or GDR states above the particle evaporation threshold. The array, which is displayed in Figure 1, is made of two detector assemblies, an inner one, which is placed downwards at backward angles with respect to the beam at $30 \mathrm{~cm}$ from the target, and an outer one, placed in the

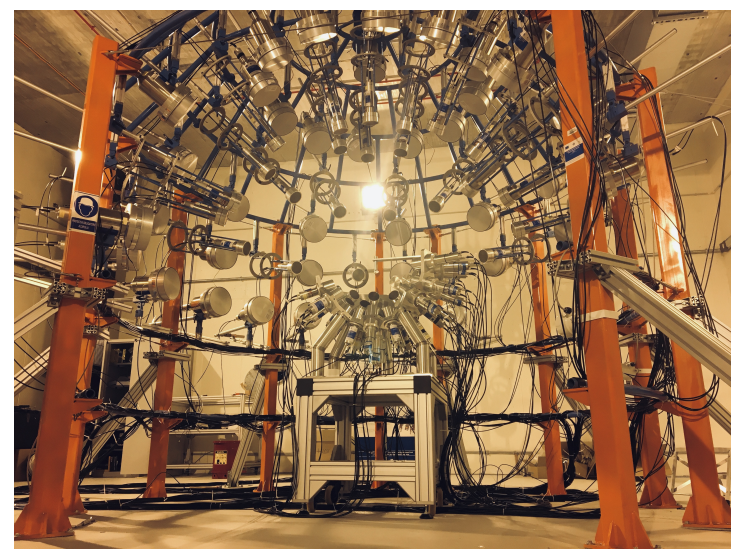

Figure 1. (Color online) The ELIGANT-GN detector in the E9 experimental hall of ELINP. Photograph courtesy of Pär-Anders Söderström. 


\section{D.L. Balabanski}

upper hemisphere at $1.5 \mathrm{~m}$ from the target. The inner array is used for $\gamma$-ray detection and consists of $19 \mathrm{CeBr}_{3}$ and $15 \mathrm{LaBr}_{3}(\mathrm{Ce})$ scintillation detectors. The outer array consists of 37 EJ-309 liquid scintillation detectors and 25 GS20 ${ }^{6} \mathrm{Li}$ glass detectors, and is used for detection of neutrons. ELIGANT-GN was commissioned with radioactive sources [23] and the performance was found to correspond to the design specifications [21] and the response simulations [22]. The energy resolution of the $\gamma$ detectors was measured with a ${ }^{137} \mathrm{Cs}$ source in the range of 3.2\%-3.6\% for the $\mathrm{LaBr}_{3}(\mathrm{Ce})$ and $4.1 \%-4.8 \%$ for the $\mathrm{CeBr}_{3}$ detectors [23]. Their time resolution is about $0.4 \mathrm{~ns}$ for the $\mathrm{LaBr}_{3}(\mathrm{Ce})$ and the $\mathrm{CeBr}_{3}$ detectors coupled to R11973 PMTs and about $0.62 \mathrm{~ns}$ for the $\mathrm{CeBr}_{3}$ detectors coupled to R6233-100 PMTs [23]. The time resolution of the EJ-309 detectors is in the range 0.4-1.1 ns, while for the GS20 ${ }^{6} \mathrm{Li}$ glass detectors it is in the range 4.2-6.8 ns [23]. The neutron energy spectrum of ${ }^{252} \mathrm{Cf}$ measured with an energy threshold of about $2 \mathrm{MeV}$ with the EJ-309 detectors, which allows to estimate an average efficiency of $2.4 \%$ for neutron energies of about 2-3 MeV [23].

\section{Studies at the Extreme of Large $N / Z$ Ratios}

One of the options for the production of neutron-rich isotopes is by utilizing photofission. This is realized at the ALTO (Accélŕateur Linéaire aurès du Tandem d'Orsay) facility at Orsay, France [10], where an linear accelerator is used to deliver a $10 \mu \mathrm{A}$ electron beam with energy of $50 \mathrm{MeV}$. The beam hits a converter and the resulting bremsstrahlung photons irradiate a ${ }^{238} \mathrm{UC}_{x}$ target. As a result, a variety of neutron-rich isotope beams are produced. Another option is to use intense quasimonochromatic beams. This possibility has not been explored yet. It has been suggested within the TDR for photofission experiments at ELI-NP [24] and for its realization proof-of-principle experiments can be carried out with the ELI-NP $\gamma$ beams.

In future, intense RIBs can be produced through photofission within the GF experiment at CERN, where very intense $\gamma$ beams will be produced. The idea is to inject a beam of partially stripped ions at the SPS and utilize laser-ion collisions for the production of $\gamma$ rays $[11,12]$. The resonant absorption of a laser photon is followed by an atomic transition. The energy of the emitted photon is boosted by the squared Lorentz factor of the relativistic ion beam. The cross section of this process is in the Gbarn range, e.g., six orders of magnitude higher compared to the cross section of LCB, which is utilized at present day $\gamma$-beam facilities, such as ELI-NP. As a result, beams with intensities of $10^{16}$ photons/s can be generated.

In a recent work, the possibility for utilization of the GF beams for studies of exotic neutron-rich nuclei, e.g., nuclei with large $N / Z$ ratios, was investigated [25]. In this case, the photons irradiate an assembly of thin self-supporting ${ }^{238} \mathrm{U}$ targets, placed in a gas cell along the beam axis and the emitted fission fragments are slowed down in the gas and extracted to form a RIB. After irradiation, fission 


\section{Research with Quasimonochromatic $\gamma$ Beams}

fragments are released kinematically from the targets, due to their large initial kinetic energy of $50-120 \mathrm{MeV}$. The fragment release is element-independent and practically instantaneous. During the slowing down process in the gas cell, the fragments are transported towards a narrow exit aperture with a combination of electric fields and during the extraction a supersonic gas jet is formed. The applied fields involves a volume DC field that drifts the heavy ions in thermal motion throughout the gas towards the wall with an exit nozzle and a surface RF field on that wall, which catches the ions above it and transports them towards the nozzle. The device which traps and transports the ions is called RF carpet [26] Optimal extraction in photofission thin-target experiments can be achieved if the drift field is applied in a direction orthogonal to the impinging $\gamma$ beam. This concept is called $\underline{H}$ igh $\underline{A}$ real $\underline{D}$ ensity with the $\underline{O}$ rthogonal extraction $\underline{C}$ ryogenic $\underline{S}$ topping $\underline{C}$ ell (HADO-CSC) [27]. It was modified for thin-target photofission experiments in Ref. [28] This device is developed in collaboration by GSI (Germany), the Giessen University (Germany), ELI-NP (Romania), Soreq (Israel) and JYFL (Finland) for the future RIB facilities at FAIR/LEB, ELISOL (RIF@IFIN) and SARAF (Soreq). Two processes are crucial for the realization of this concept, i.e., the proper handling of the ion transport in gas jets and at the RF-carpet. The estimated isotope yields after extraction from the HADO-CSC, i.e. before mass separation, are presented in Figure 2. The path of the astrophysical $r$-process after Ref. [29] is shown in the figure. The calculations indicate that RIB production with the GF $\gamma$ beams at CERN will provide an opportunity for detailed studies of neutron-rich nuclei in the vicinity of the $N=50$ and $N=82 r$-process waiting points.

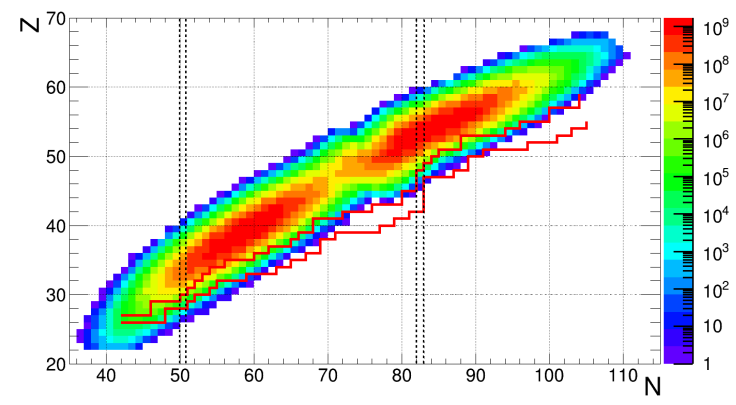

Figure 2. (Color online) Estimated yields of neutron-rich isotopes, produced in photofission with the GF $\gamma$ beams. The red lines indicate the $r$-process path [29].

\subsection{Towards a HADO-CSC prototype at ELI-NP}

The CSC for the proposed IGISOL beamline at ELI-NP has several features which differ from other existing gas stopping cells, e.g., a primary $\gamma$ beam, a large number of targets in a large gas volume, an orthogonal DC electric field coupled to multiple RF fields applied on the carpet, and a dual-chamber design. 


\section{D.L. Balabanski}

The primary gas chamber, where the target assembly is placed, is at a He gas pressure in the range of 200-300 mbar, which is needed to efficiently thermalize the fission fragments. It communicates with the upper chamber, which is at a lower pressure of several mbar, via a number of apertures with a diameter of about $1 \mathrm{~mm}$. The upper walls of both chambers should be covered by RF carpets which will catch and guide the ions towards the exit nozzles. The electrode structure surrounding the target area creates a uniform electric field which pushes the thermalized fragments towards the upper-wall carpet.

The transversal extraction through multiple nozzles generates a complex gas flow through the entire cell, which requires a detailed study with a software dedicated to computational fluid dynamics, such as COMSOL [30]. The gas enters the lower chamber through a bottom inlet and exits the upper chamber through the exit nozzles and one or two outlets on its sides. An extra outlet is used in the lower chamber to better control the gas parameters. The pressure in the upper chamber is created by gas jets which are injected through the nozzles. The gas leaves this chamber though a single exit, where the extracted ions are injected in a radio-frequency quadrupole (RFQ) system, where a beam is formed. Differential pumping is applied, such that vacuum is created in RFQ. The gas velocity distribution in the entire CSC with a pressure of $300 \mathrm{mbar}$ in the lower chamber and of $10 \mathrm{mbar}$ in the upper chamber, at a temperature of $70 \mathrm{~K}$, has been calculated [31] and an operational regime has been suggested.

A test bench for studies of supersonic gas jet dynamics has been assembled at ELI-NP. It consists of a transparent two-chamber gas cell. The He gas penetrates in the second chamber through a small nozzle forming a gas jet. The jets are imaged with the Schlieren photography method. The velocity of the gas in different parts of the jet is mapped with a Pitot tube. The temperature of the jet is also monitored. Flanges with nozzles having different sizes can be mounted between the two chambers. These test measurements are done at room temperature and aim at validation of the model calculations [31,32]. Nevertheless that the CSC will be operational at a temperature of $70 \mathrm{~K}$, the measurements, which are ongoing, will provide a solid ground to choose the optimal nozzle size. A photograph of the experimental setup is shown in Figure 3.

The ion transport towards the extraction nozzles of the HADO-CSC is done with RF carpets. They cover the walls where the exit apertures are placed. Electromagnetic fields are applied on the RF carpets for trapping the ions, preventing their adhesion to the wall, and transporting them towards the exit nozzles. An RF field is used for ion capture and a radial DC field for ion transport. At lower gas densities, the ion transport is achieved using the traveling-wave technique $[33,34]$. A critical parameter for the ion-transport efficiency of the RF carpet is the density of electrodes. A novel RF carpet design, using spiral electrodes, was suggested, which enables the construction of RF carpets with very high electrode density. The new carpet performance was validated using ionoptics simulations and was compared to classical RF carpets, which use con- 


\section{Research with Quasimonochromatic $\gamma$ Beams}

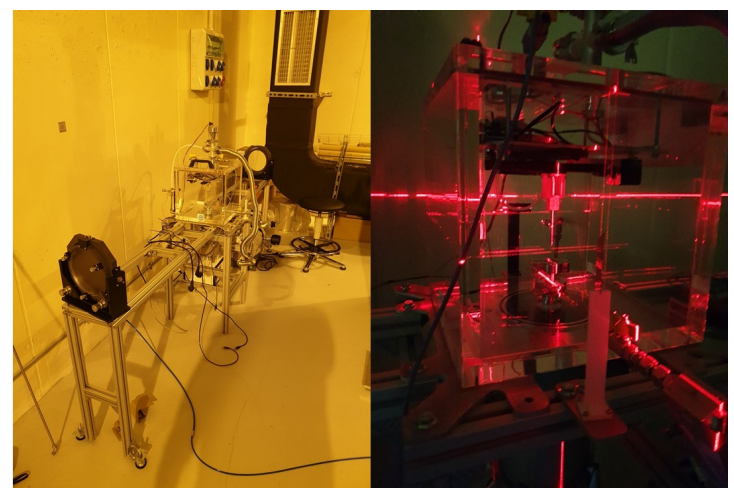

Figure 3. (Color online) Experimental setup for gas-jet imaging with the Schlieren photography method. A transparent gas chamber is placed between two 8 inch mirrors mounted on precision gimbal mirror mounts (left). Focused light is used for gas-jet imaging (right). Photograph courtesy of Alexandru State.

centric rings [35]. A test bench for studies of RF-carpet ion transport is being assembled at present at ELI-NP.

\section{Towards $\gamma$-Beam Experiments with OAM $\gamma$-Ray Beams}

An important new development in the field of nuclear photonics is the production and utilization of twisted photons. Experiments for generation and utilization of OAM photons have been initiated and successfully carried out in solidtarget experiments at the Ohio State University [36] and at the $1 \mathrm{PW}$ beamline of the ELI-NP high-power laser system (HPLS). The generated vortex laser pulses were used for acceleration of protons. The analysis of these data is ongoing. Further experiments are carried out with a gas target, aiming at the verification of the generation of ultrarelativistic monoenergetic electron bunches via a twisted laser, which was described theoretically recently [37].

While the production of high-power laser pulses is rather straightforward and has been realized in different laboratories, the generation of high-energy twisted photons is an open problem, since it involves the transfer of the orbital angular momentum in Compton backscattering. The process is predicted theoretically $[38,39]$. However, there are approaches, which find out that the photon angular momentum cannot be transferred in Compton scattering [40]. This issue still awaits its experimental verification. Such experiments are planned at the ELI-NP HPLS in early 2022.

Experiments with OAM $\gamma$ rays will open a whole variety of research opportunities in nuclear structure physics. Since there are not a single facility in the world where vortex $\gamma$ rays can be generated, ongoing research is focused on the- 


\section{D.L. Balabanski}

ory. As a first step, the twisted photons were described in spherical waves [41]. The modification of the photonuclear reaction cross sections in experiments with twisted photons were evaluated [42], as well as the generalization of the NRF formalism for higher angular momenta of the incident photons and possible experimental applications are studied.

\section{Studies of Photonuclear Reactions at ELI-NP}

Photodisintegration is one of the basis research techniques, which will be used at ELI-NP. Experimental setups for measurements of photoneutron and chargedparticle reactions were built and are ready to take the first beams, which are expected to be delivered in 2023. The experimental techniques, the observables and the quantities, which are deduced in these experiments, were reviewed in Ref. [1]. The expected increase of the of the spectral density of the $\gamma$ beams at ELI-NP with about two orders of magnitude will bring the experimental techniques to a new level of sensitivity. The experimental program at the facility, which has been described recently by Tanaka et al. [43], includes studies related to nuclear astrophysics and precise measurements of photoneutron cross sections.

Photonuclear reactions are one of the mechanisms involved in the nucleosynthesis. Photons from the high-energy tail of the Plank distribution can photodisintegrate nuclei. This is demonstrated best in the synthesis of nuclei heavier than iron via the $p$ process, where $(\gamma, \mathrm{n}),(\gamma, \mathrm{p})$ and $(\gamma, \alpha)$ reactions are one of the driving forces [44]. Another example, which underlines the importance of photonuclear reaction studies in astrophysics is related to studies of time-reverse reactions, such as e.g., ${ }^{16} \mathrm{O}(\gamma, \alpha){ }^{12} \mathrm{C}$ reaction. In this experiment, due to the low reaction cross section close to the Gamow window, a time projection chamber (TPC), with optical readout, i.e., an active target, was used [45].

In active-target experiments, the target, which is a gas, serves also as a detector medium. In the photonuclear reaction drifting electrons are created in the active volume of the TPC. At ELI-NP, two active-target experimental setups based on the time projection chamber (TPC) technology will be available, the miniTPC and the ELITPC [46]. The mini-TPC is available for experiments and the ELITPC is still under implementation. Both of them use an electronic readout based on the GEM detector technology [47,48]. The difference between the two detectors is their active volume and number of readout channels. The mini-TPC utilizes a 256-channel readout in the horizontal plane, perpendicular to the drifting electrons. It consists of three layers of electrodes, refered as $u-v-w$ readout. They are oriented at $120^{\circ}$ to each other and placed on a multilayer PCB. The ELITPC will utilize a 1024-channel readout. The signals form the readout are processed by GET (General Electronics for TPCs) boards [49].

In addition, a $4 \pi$ array of Si strip detectors, the ELI-NP Si Strip Array (ELISSA) 


\section{Research with Quasimonochromatic $\gamma$ Beams}

is available for charges-particle experiments at ELI-NP [46]. It consists of 36 X3-type double-sided Si strip detectors (DSSSD), which form a barrel, and eight QQQ3-type DSSSDs, which cover the endcups. The barrel is built and tested and the endcups are still to be implemented. The total angular coverage of the instrument will be $80 \%$, and it will detect protons in the energy range of $E_{p}=$ $100 \mathrm{keV}-10 \mathrm{MeV}$ and $\alpha$ particles of $E_{\alpha}=100 \mathrm{keV}-30 \mathrm{MeV}$.

A proof-of-principle experiment was carried out at the $\mathrm{HI} \gamma \mathrm{S}$ facility, Duke University [50]. There the photodisintegration of ${ }^{7} \mathrm{Li}$ was studied [51], which is of importance to understand the Big Bang nucleosynthesis. For this purpose, monoenergetic photons in the energy range 4.4 to $10 \mathrm{MeV}$ have been used, and tritons and $\alpha$ particles were detected in coincidence by segmented Si detectors.

\subsection{Studies of photoneutron reaction cross sections}

Photoneutron reaction cross-section data are used in a variety of applications, e.g., physics and technology of fission reactors, radiation-shielding design, safeguards, nuclear-waste transmutation, medical-isotope production, calculations of absorbed dose in human body during radiotherapy, nuclear astrophysics, etc. An open problem in the field are the discrepancies in results from different measurements. In the last decades, IAEA organized two coordinated reserch projects to eveluate the total $(\gamma, \mathrm{n})$ and the partial $(\gamma, \nu \mathrm{n}), \nu=1,2,3 \ldots$ cross sections. The last one resulted in the 2019 IAEA Photonuclear Data Library [52]. Although that the cross sections for more than 200 isotopes were re-evaluated, and in some cases additional measurements were done with LCB beams [52], the problem is not resolved yet, since discrepancies persist even in data obtained in experiments with LCB beams carried out in different laboratories [53]. The problem is related to the fact that the experiments are done with different detectors and different beam diagnostic devices. Different data reduction techniques are used, too.

Nowadays, the flat-efficiency detector technique [54] is used for measurements of photoneutron cross sections. It aims at direct neutron multiplicity determination in nuclear reaction measurements with neutron emission. The neutron proportional counters are placed in such way in a moderation material that the detection efficiency does not depend on the neutron kinetic energy. A flatefficiency detector, called ELIGANT-TN, where TN stays for 'thermalized neutrons', was developed at ELI-NP. Its $28{ }^{3} \mathrm{He}$ proportional counters are placed in three concentric rings in a neuron moderator polyethylene cube. The detector is designed such that it has flat efficiency of about $40 \%$ for neutron energies up to $5 \mathrm{MeV}$ [55].

The UHECR propagation models require a good knowledge of the total photoabsorption cross section for at least the most abundant complex nuclei in the universe $\left({ }^{4} \mathrm{He},{ }^{12} \mathrm{C},{ }^{16} \mathrm{O},{ }^{20} \mathrm{Ne},{ }^{24} \mathrm{Mg},{ }^{28} \mathrm{Si}, \ldots{ }^{56} \mathrm{Fe}\right)$, ideally for around 380 isotopes up to ${ }^{56} \mathrm{Fe}$. In light nuclei the predicted GDR peak energies and cross 


\section{D.L. Balabanski}

sections, which are calculated with present-day nuclear models, e.g., TALYS1.95 [56], can vary by large factors, which can induce significant changes in the UHECR propagated spectra. Three facilities are involved in the PANDORA ( $\underline{P}$ hoto- $\underline{A}$ bsorption of $\underline{N}$ ucleai and $\underline{D}$ ecay $\underline{O}$ bservation for $\underline{R}$ eactions in $\underline{A}$ strophysics) project. In studies at the RCNP laboratory of the University of Osaka (Japan) and iThemba LABS, Capetown (South Africa) virtual photons will be used, via Coulomb excitation induced by high-energy proton scattering $\left(E_{p}>200 \mathrm{MeV}\right.$ ), to extract the total $E 1$ strength distribution up to $32 \mathrm{MeV}$ and $24 \mathrm{MeV}$, respectively. Experiments at ELI-NP will make use of high-flux quasimonoenergetic real photon beams with very high-energy resolution $(<0.5 \%$ FWHM) to measure the absolute photoabsorption cross section up to $20 \mathrm{MeV}$.

\section{Summary and Outlook}

The presented overview of the research work in the field of nuclear photonics with intense $\gamma$ beams at ELI-NP demonstrates the vitality of the field. The expected launch of the $\gamma$-beam facility will boost research in the field. For this purpose, a variety of state-of-the-art instruments were constructed. Other developments are related to studies of exotic nuclei produced in photofission. These open a niche for a RIB facility at IFIN-HH (RIF@IFIN), and in future, within the GF experiment at CERN. Last, but not least, the possibility to generate highenergy OAM photons will open a completely new experimental field.

\section{Acknowledgements}

This work is supported in part by Grant No. PN-III-P4-ID- PCCF-2016-0164 of the Romanian National Authority for Scientific Research and Innovation.

\section{References}

[1] A. Zilges, D.L. Balabanski, J. Isaak, N. Pietralla (2022) Prog. Part. Nucl. Phys. 122 103903 DOI: https://doi.org/10.1016/j.ppnp.2021.103903.

[2] R.H. Milburn (1963) Phys. Rev. Lett. 1075.

[3] F.R. Arutyunian, V.A. Tumanian (1963) Phys. Lett. 4176.

[4] H. Ohgaki et al. (1994) Nucl. Imstrum. Meth. Phys. Res. A 353384.

[5] N. Pietralla et al. (2002) Phys. Rev. Lett. 88012502.

[6] M. Bhike, W. Tornow, Krishichayan, A.P. Tonchev (2014) Phys. Rev. C 95024608.

[7] Krishichayan et al. (2019) Phys. Rev. C 100014608.

[8] A. Göök et al. (2011) Nucl. Phys. A 8511.

[9] A. Göök et al. (2017) Phys. Rev. C 96044301.

[10] F. Azaiez, S. Essabaa, F. Ibrachim, D. Verney (2013) Nucl. Phys. News 23:2 5.

[11] M.W. Krasny (2015) The Gamma Factory Proposal for CERN. arXiv:1511.07794 [hep-ex]. 
[12] M.W. Krasny (2019) "Gamma Factory Proof-of-Principle experiment" (CERNSPSC-2019-031).

[13] Yijie Shen et al. (2019) Light Sci. Appl. 890.

[14] M.B. Chadwick et al. (2003) Nucl. Sci. Eng. 144157.

[15] M.C. White et al. (2003) Nucl. Sci. Eng. 144174.

[16] E. Khan et al. (2005) Astropart. Phys. 23191.

[17] R. Alves-Batista et al. (2015) J. Cosmol. Astropart. Phys. 10063.

[18] C.A. Ur et al. (2016) Rom. Rep. Phys. 68 S483.

[19] P.-A. Söderström et al. (2019) Acta Phys. Pol. B 50329.

[20] L. Capponi et al. (2021) JINST 16 T12002.

[21] F. Camera et al. (2016) Rom. Rep. Phys. 68 S539.

[22] M. Krzysiek et al. (2019) Nucl. Inst. Methods Phys. Res. A 916257.

[23] P.-A. Söderström et al. (2021) Nucl. Inst. Methods Phys. Res. A in print.

[24] D.L. Balabanski et al. (2016) Rom. Rep. Phys. 68 S621.

[25] D. Nichita et al. (2021) Ann. Phys. 2100207.

[26] M. Wada et al. (2003) Nucl. Inst. Methods Phys. Res. B 204570.

[27] T. Dickel et al. (2016) Nucl. Inst. Methods Phys. Res. B 376216.

[28] P. Constantin et al. (2017) Nucl. Inst. Methods Phys. Res. B 3971.

[29] H. Schatz, K. Blaum (2006) Europhys. News 37:5 16.

[30] (accessed on Oct. 15, 2021) http://www.comsol.com.

[31] D.L. Balabanski et al. (2021) JPS Conf. Proc. 35011010.

[32] A. State et al. (2020) UPB Sci. Bull. A 82:2 197.

[33] G. Bollen et al. (2011) Int. J. Mass Spectrom. 299131.

[34] F. Arai et al. (2015) Int. J. Mass Spectrom. 36256.

[35] A. Rotaru et al. (2021) Int. J. Mass Spectrom. submitted.

[36] M. Cernaianu et al. (2021) in preparation.

[37] Yin Shi, D. Blackman, D. Stutman, A. Arefiev (2021) Phys. Rev. Lett. 126234801.

[38] U.D. Jentschura, V.G. Serbo (2011) Phys. Rev. Lett. 106013001.

[39] T. Maruyama, T. Hayakawa, T. Kajino (2019) Sci. Rep. 951.

[40] S. Stock et al. (2015) Phys. Rev. A 92013401.

[41] V. Baran et al. (2021) Phys. Rev. A submitted.

[42] Yi Xu, D.L. Balabanski (2021) in preparation.

[43] K.A. Tanaka et al. (2020) Matter Radiat. Extremes 5024402.

[44] M. Arnould, S. Goriely (2003) Phys. Rep. 3841.

[45] R. Smith et al. (2021) Nature Commun. 125920.

[46] O. Tesileanu et al. (2016) Rom. Rep. Phys. 68 S699.

[47] F. Sauli (1997) Nucl. Instrum. Methods Phys. Res. A 386531.

[48] F. Sauli (2016) Nucl. Instrum. Methods Phys. Res. A 8052.

[49] E. Pollacco et al. (2012) Phys. Procedia 371799.

[50] H.R. Weller et al. (2009) Prog. Part. Nucl. Phys. 62257.

[51] M. Munch et al. (2020) Phys. Rev. C 101055801.

[52] T. Kowano et al. (2020) Nucl. Data Sheets 163109.

[53] A. Banu et al. (2019) Phys. Rev. C 99025802.

[54] A. Tarifeno-Saldiva et al. (2017) JINST 12 P04006.

[55] M. Krzysiek et al. (2019) AIP Conf. Proc. 2076040004.

[56] A. Koning, D. Rochman (2012) Nucl. Data Sheets 1132841. 